\title{
Warranty of Misinforming as an Option in Product Utilization Process
}

\author{
Dimitar Christozov \\ American University in \\ Bulgaria, Blagoevgrad, \\ Bulgaria \\ dgc@aubg.bg
}

\author{
Stefanka Chukova \\ Victoria University of \\ Wellington, \\ New Zealand
}

stefanka.chukova@vuw.ac.nz

\author{
Plamen Mateev \\ Sofia University "St. Kliment Ohridski”, Sofia, Bulgaria \\ pmat@fmi.uni-sofia.bg
}

\begin{abstract}
The following definition of "option" is given in Wikipedia - "In finance, an option is a contract, which gives the buyer (the owner or holder) the right, but not the obligation, to buy or sell an underlying asset or instrument at a specified strike price on or before a specified date, depending on the form of the option" ("Option," n.d.). Option as a risk management (mitigation) tool is broadly used in finance and trade. At the same time, it introduces asymmetry in the sense that, probabilistically, it limits the level of losses (e.g., the price of the option) and allows for unlimited gains. In the market of sophisticated devices (as smart phones, tablets, etc.), where technologies are rapidly advancing, customers usually do not have the experience to use all features of the device at the time of the purchase. Due to the lack of appropriate expertise, the risk of misinforming, leading to not purchasing the "right" device is high, but given enough time to learn the capabilities of the device and map these to the needs and tasks that device will be used for, could provide the client with substantial long term benefits. Warranty of misinforming is a mechanism that provides the client with the opportunity to explore the device and master its features under limited risk of financial losses. Thus, the warranty of misinforming could be considered as an option - the customers buy it (at a fixed cost) and may gain (theoretically) unlimited benefit by realizing (within the terms of the warranty) that the device can be used to solve a variety of problems not envisaged at the time of purchase. In this study we present the idea of treating the warranty of misinforming as an option in finances and provide examples to illustrate our viewpoint.
\end{abstract}

Material published as part of this publication, either on-line or in print, is copyrighted by the Informing Science Institute. Permission to make digital or paper copy of part or all of these works for personal or classroom use is granted without fee provided that the copies are not made or distributed for profit or commercial advantage AND that copies 1) bear this notice in full and 2) give the full citation on the first page. It is permissible to abstract these works so long as credit is given. To copy in all other cases or to republish or to post on a server or to redistribute to lists requires specific permission and payment of a fee. Contact Publisher@InformingScience.org to request redistribution permission.
Keywords: warranty of malfunctioning, warranty of misinforming, options, asymmetry, utility, risk of misinforming.

"Trial and error is freedom." (Taleb, 2014, p. 246) 


\section{Introduction}

The market of complex and technologically advanced devices is growing quickly, offering many challenges to all involved parties. The major challenge is that many new features are included in the next generation devices and these new devices show up on the market almost on a daily basis. This quick turnaround does not allow the customers to learn how to fully utilize the device's built-in features. Additionally, the smart devices allow an easy extension of their capabilities by adding a variety of on-line applications.

All of the above significantly affect the risk of misinforming. Usually, at the time of purchase, the seller is not familiar with the problems/tasks the costumer aims to solve with the device and therefore is not able to provide in depth advice. The customer has to gain experience in using the device and mapping its features to the problems/tasks s/he faces. Also, often it is difficult to realize that a newly encountered problem/task can be addressed by the same device as the customer was not aware of this possibility at the time of purchase and, of course, s/he had not included those in her/his purchasing motivation.

This shows that there might be also some positive outcomes of misinforming. The option of having positive effect of misinforming has not been emphasized in any earlier studies and definitely deserves some attention. When it comes to these positive outcomes, the information asymmetry between the seller and buyer is not critical for quantifying the risk of misinforming, and moreover the information asymmetry cannot be reduced by improving the communication process between these two parties. In addition, even clients' training would not necessarily reveal the hidden potential of the product and reduce the misinforming. The full benefit of the device for a particular client is usually understood, often by chance, via trials and errors and it is highly dependent on client's ability to adopt quickly the incoming technical advances for the device.

Nowadays purchasing decisions have to incorporate a new source of uncertainty: whether to buy a more advanced and expensive device, or to limit the purchase to a device that will allow accomplishing only the set of tasks, as identified at the time of purchase? In other words, the client is facing the following dilemma: buying a low price device with limited opportunity for future benefits vs. paying a high price for a device with unknown potential benefits. This is a typical scenario that motivates options launching in trade and finance.

The warranty of misinforming could be interpreted as an option in product purchasing: within a low risk environment, it provides the clients with the chance of trying and experimenting with the device, i.e., with some learning opportunities regarding the device. The cost of this "option" is the learning time - time spent to explore the device, plus the cost of purchasing the warranty. Depending on the warranty policy the latter cost might be refundable.

The concept of misinforming, as an incorrect interpretation on behalf of the client of otherwise correct information provided by the sender, is well defined within Informing Science literature (see for example E. Cohen, 2007, and the book "Foundation of Informing Science", edited by Eli Cohen and Grandon Gill, 2009). Warranty as a factor for informing quality is introduced by Zbignew Gackowski (2007). Studies on different aspects of how warranty may affect the risk of misinforming are presented in numerous of our publications (Christozov, Chukova, \& Mateev, 2014). We define "the warranty of misinforming", within the frame of Informing Science, as the tool providing the client a "risk free" period to verify the usefulness of received information within the context of faced problems. The warranty of misinforming is especially applicable within a commercial Selling-Buying transaction in acquiring a new product. So far, we had studied the negative effect of misinforming. In this study we address the positive impact of warranty of misinforming. 
To illustrate the above idea, let us consider the case of buying a smart phone. The initial need is to obtain a device that will allow the client to make calls and to exchange text messages. The customer has many options, from relatively cheap to extremely expensive devices, which allow $\mathrm{him} / \mathrm{her}$ to accomplish these tasks. Let us assume also that the price corresponds to device's properties (e.g., the size of the screen). After buying a smart phone, the client discovers that there is a huge set of applications, developed by various entities, available on-line. Installing an appropriate application enriches the device's capability to solve different problems/tasks. Let us assume that the customer has installed an application, which allows for the smart phone to be used as a GPS navigation system and that the business of the client includes driving in unknown areas delivering goods. The new navigation system will increase the client's productivity by decreasing the time needed to locate the required destinations. Unfortunately, the client realizes that $\mathrm{s} / \mathrm{he}$ had not bought the best device to address this task, because the device's screen is too small. At the time of purchase the screen was chosen according to the initially recognized needs (Figure 1) and the newly added task of "navigation" was not taken into account.

Typically, the warranty of misinforming provides the client with a "free" trial period for familiarizing himself/herself with the device. The price client pays for this "free" trial period is the charge for the warranty and the time spent learning the features and capabilities of the device. In the case of a smart device, its capabilities can be supplemented by apps and add-ons produced by a huge number (millions) of vendors. Obviously, the primary producer of the device is not in a position of comprehensively "informing" the clients about these additional capabilities. Moreover, at the purchase time even the client does not know what will be the most beneficial usage of the devices. In the above example, the navigation property of a smart phone turned out to be very beneficial for our specific customer. This additional utility of the device has been discovered by learning and installing an appropriate application well after the initial purchasing decision.

Therefore, based on the above comments, we can conclude that purchasing a warranty of misinforming for a device could be considered to be equivalent to purchasing an option in financial context. One may decide to purchase more complex and technically advanced device with better properties and higher quality (e.g., larger screen) and to explore it during the trial period, to learn whether it can be beneficial for accomplishing tasks beyond the originally recognized set of tasks. In this example "the navigation" is an extra task, beyond the initial set of tasks of "making calls and exchanging text messages".

This study maps the option risk mitigation principles to the risk of misinforming and explores the specific properties of warranty of misinforming when considered as an option. In the next section (Background) the foundations related to the risk of misinforming and option principles are presented from warranty of misinforming as an option viewpoint. The section "The Model" maps warranty of misinforming as an option to risk of misinforming, discussing the sources of costs and benefits. In general, we elaborate on the following idea: the warranty of misinforming for a smart device has pre-specified limited cost, but advanced utilization of the device may bring unlimited potential benefit.

Further, we discuss the case of "warranty of misinforming" as an option from several different aspects. It is well known that the clients' main benefit from this warranty is the opportunity to study the features of the device with no risk. In addition, the learning opportunities are not limited only to the properties of the device, but extend to mapping the client's problems/tasks to solutions enhanced by the available technical advances for the device, e.g., apps, add-ons, etc. Identifying such learning opportunities is the main effect of positive misinforming: firstly, the buyer underestimates the potential of the device, and secondly, while the buyer utilizes the product to solve his/her initial set of problems/tasks, s/he realizes that the product - the smart device - is suitable to solve problems/tasks of larger scale than initially thought. 
The paper enriches the understanding of warranty of misinforming as a factor for enhancing informing quality not only by reducing the negative aspects of misinterpretation, but also by serving as a learning tool for acquiring more comprehensive understanding of the meaning and impact of provided message.

\section{Background}

\section{Information Asymmetry}

What is the main reason for the existence of information asymmetry? Information asymmetry is due to the communication process environment:

1. The seller is an expert on the features, specification, and properties of the product/device.

2. The client has a limited expertise on the properties of the device and has a set of prespecified problems/tasks that has to be solved by using the device.

3. The communication process between the seller and buyer does not fully reveal the properties of the device during the sale process, i.e., the expertise mismatch of the two parties leads to

- a positive purchase decision - buying a device that is not suitable for client's tasks, or

- a negative purchase decision - not buying a perfectly suitable for client's tasks device.

This is a two-sided information asymmetry, which typically can be reduced by improving the communication process. For more see Christozov, Chukova, and Mateev (2009, 2011, 2014). In the case of the product being a smart device, usually there is a list of client's problems/tasks that were not related to the product, and even more, were not targeted by the producer at the product design phase. These additional problems/tasks are not identified and communicated with the producer or supplier prior to the purchase. It is well known that the usage of smart devices in carrying out specific tasks is highly individual and in general it might be impossible for the producer to envisage all potential usages of the device for solving the client's additional tasks.

Finally, there are two types of possible contributions in "producing" smart devices: (1) producing the device itself (such as Apple, Samsung, etc.) and (2) developing applications for the device (theoretically everyone may develop and promote his/her smart device application on the market). This makes the task of reducing information asymmetry via improving communication between the parties involved in the purchase practically unsolvable. Because everyone with appropriate skills may develop an application, and most of these applications are easily available on-line, the actual number of "producers" is unlimited and a direct communication with all of them is impossible.

\section{Options - Risk Asymmetry: Positive vs. Negative Misinforming}

So far, in our earlier studies, we focused on the negative aspects of misinforming (Christozov et al., 2014). Here, in this paper, we elaborate on the possible positive aspects of misinforming. Due to misinforming, it is possible that the client buys a product with much richer set of functionalities and/or properties with usage opportunities that go well beyond the initial set of problems intended to be solved with the product. This positive aspect of the information asymmetry reveals itself during the learning process in the utilization phase of product adoption. One can buy a product to address a set of particular needs, but it may turn out (during product adoption) that the product is suitable for solving a set of client's additional advanced tasks. The client was not aware of these additional, advanced tasks at the time of the product purchasing, or the client was 
not aware that these type of problems can be solved by using the device - overall, the benefit from product's full utilization may dramatically exceed (theoretically/potentially unlimited) the expectations of the product initial utilization, which were the motivation for the positive purchase decision.

Option, as a tool to mitigate the market risks, has the following important property - it is risk asymmetric - a fixed loss, which is the price to purchase the option and (theoretically/potentially) with unlimited gain. The potential gain, in case of misinforming, requires learning by trying and experimenting. Also, it requires an expertise to recognize that a problem can be solved with the particular device, or if the problem cannot be solved with the available device what type of device is needed to target the problem successfully. Taleb (2014) elaborates:

"... all we need is the ability to accept that what we have on our hands is better than what we had before-in other words, to recognize the existence of the option (or "exercise the option" as people say in the business, that is, take advantage of a valuable alternative that is superior to what precedes it, with a certain gain from switching from one into the other, the only part of the process where rationality is required). And from the history of technology, this ability to use the option given to us by antifragility is not guaranteed: things can be looking at us for a long time." (p. 190)

"Trial and error has one overriding value people fail to understand: it is not really random, rather, thanks to optionality, it requires some rationality. One needs to be intelligent in recognizing the favorable outcome and knowing what to discard.” (p. 192)

"Trial and error is freedom." (p. 246)

The warranty of misinforming provides a risk free trial and error period and encourages exploring and discovering product's capabilities.

The smart devices market is an environment, where the positive aspect of the risk of misinforming can be easily observed. For example, one is buying a new smart phone, due to say battery failure of the old one, expecting to use it only to make calls and send/receive text messages, but ends up with a device having much broader list of functionalities (Figure 1) useful to support everyday activities.

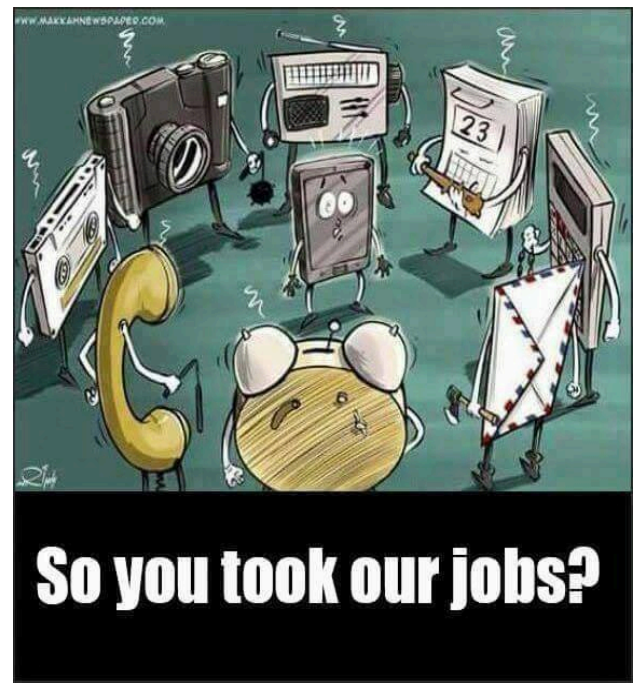

Figure 1. Smartphone jobs

Downloaded from: http://fastler.net/p/0q1 


\section{Related Works}

We have made an extensive search in a number of databases, such as SCOPUS, EBSCOhost (which by itself includes more than 40 databases), and Emerald, using different combinations of keywords, like "warranty" and "options (or "securities", or "finance options") aiming to provide an overview of literature related to our problem. Our repeated searches led consistently to empty outcome listings. Based on these results and to the best of our knowledge the concept presented here offers a novel interpretation of the warranty of misinforming. On the other hand, the database search led us to several studies on the relationship between warranty and information asymmetry, which we found useful in our work and which we provide below.

In Dewally and Ederington (2006), the authors consider signaling strategies that sellers of higherquality products or securities employ to differentiate their products. They study four of these strategies: (1) development of a reputation for quality, (2) third-party certification, (3) warranties, and (4) information disclosure. Using data from the online auction market for classic comic books they show that for this market the information asymmetry is substantial and the listed signaling strategies are common, i.e., warranty could be used as a mitigation tool for information asymmetry.

Nazari and Arab (2014) consider information asymmetry between buyers and manufacturers and the role of external signals, such as price, brand, advertising, packaging, country of origin, previous experience, price discounts, hardware features and warranty, on the quality perception and purchase intention of consumers. They use laptop as a case study and show that warranty has a significant effect on clients' quality perception as well as purchase intention.

Wankhade and Dabade (2006), focused on the information asymmetry in business processes and its effect on the market dynamics. They show that the advertising, word-of-mouth, rebate, warranty and guarantee, mitigate the effect of information asymmetry on quality perception.

\section{The Model}

The model describes the following categories (entities) and the relationships between them:

\section{Product}

A product $D$ (device) is described by a set of properties, $P=\left\{\left\{p_{i}, v_{i}\right\}, i=1,2 \ldots, k\right\}$, where every property is described by a pair $p_{i}$ - the name of the property, and $v_{i}$ - numerical descriptor/value. For example, the property screen size is presented as \{"screen size", 5 inches\}.

\section{Client}

A client $C$ is described by a set of needs (problems/tasks), s/he intends to solve/accomplish by using a product of a particular type. This set of needs motivates the client to buy the product $D$, which provides the best option in satisfying her/his needs:

- The purchase of the product $D$ has been motivated by the initial set of needs $N_{0}$ - a set of problems/tasks the client faces and wants a device/product, which will allow her/him to solve them. To accomplish his/her tasks, the product must possess a set of properties $P_{N_{0}}^{c}=\left\{\left\{p_{i}, v_{i}\right\}, i=1, \ldots k_{N_{0}}^{c}\right\}$, that satisfy the minimal level of acceptance (quality) $Q_{N_{0}}^{c}=\left\{\left\{p_{i}, q_{i}^{\text {min }}\right\}, i=1, \ldots, k_{N_{0}}^{c}\right\}$. (Example: If the client needs a phone to make calls and to send/receive SMS: (1) the property "screen size" is relevant, and (2) the minimal level of acceptance - the minimal value of the property "screen size" - is 2 inches.)

- After the purchase of the product, a trial period starts and the set of needs evolves, so that they became $N_{t}$ after a trial period of length $t$. The set $N_{t}$ : is an extended set of prob- 
lems/tasks, identified by the client during the trial period, that are (1) recognized as needed and (2) potentially solvable with a product of the given type. The set $N_{t}$ is not necessarily solvable with the purchased product, because the values of its properties do not satisfy the quality requirements (minimal acceptance levels) of the new needs. For example: the purchased smart phone allows for navigation via GPS, but to make practical use of it, the property - screen size - must be at least 5 in, whereas the purchased product has a screen size of 2 in.

\section{Applications}

Consider the set of applications $A=\left\{\left\{a_{j}, P^{a_{j}}, Q^{a_{j}}\right\}, j=1,2, \ldots n\right\}$, which includes additional software tools (not provided by the producer at the time of purchasing), developed by different vendors, that are available on-line by producer's store and can be installed on the product to enrich its usability.

Suppose that an application $a$ requires a set of properties of the product $P^{a}=\left\{p_{i}^{a}, v_{i}^{a}\right\}$ with qualities $Q^{a}=\left\{p_{i}^{a}, v_{i}^{a} \geq q_{i}^{\text {min }}\right\}$. For applications, quality means the level of usability expressed by the property value. (For example: On a smart phone with a 2-inch screen one can run navigation software, but its practical use while driving is very low - say $10 \%$. For the same smart phone, but with 5 inches screen, the usability could be much higher - say 90\%.)

\section{Process}

The process would follow two phases, namely that of purchasing then learning, as shown in Figure 2 (illustration of the process of learning), and elaborated as follows:

1. Phase - purchasing: The purchasing decision is made according to $N_{0}$. We make the following assumptions:

- (A1) the price of the product resembles the product quality level, which represents the product properties, unrelated to a specific client,

- (A2) the client tends to minimize the price, i.e., chooses a product that satisfies $Q_{N_{0}}^{c}$ on minimal level of $v_{i}$ of relevant properties.

The client makes a decision based on subjective assessment of product's properties $\widehat{P_{0}}(D)=$ $\hat{P}\left(m\left(P_{D}\right)\right)$, as understood from $m\left(P_{D}\right)$ - the message describing the product provided by the producer.

2. Phase - learning: The learning includes "trial and error" time during a period $t$. It is identified by the triple $\left(\widehat{P_{t}}, \widehat{A_{t}}, \widehat{N_{t}}\right)$ function. This phase discovers what the product allows the client to do with it. This may require reassessing the set of relevant product properties and related levels of qualities - $\widehat{P_{t}(D)}$. Also, a subset $\widehat{A_{t}}$ of available applications $A$ might be tested by the client during the trial period $t$ and recognized as useful. This testing might result in adding problems that were not initially considered as appropriate to be attempted with the product $-\widehat{N_{t}}$. During this phase, the client may learn about applications, which would require properties at a higher quality than currently available, and adjust his/her purchasing criteria (e.g., to gain full benefit from the navigation application, the size of a smart phone screen must be at least 5 inches). 


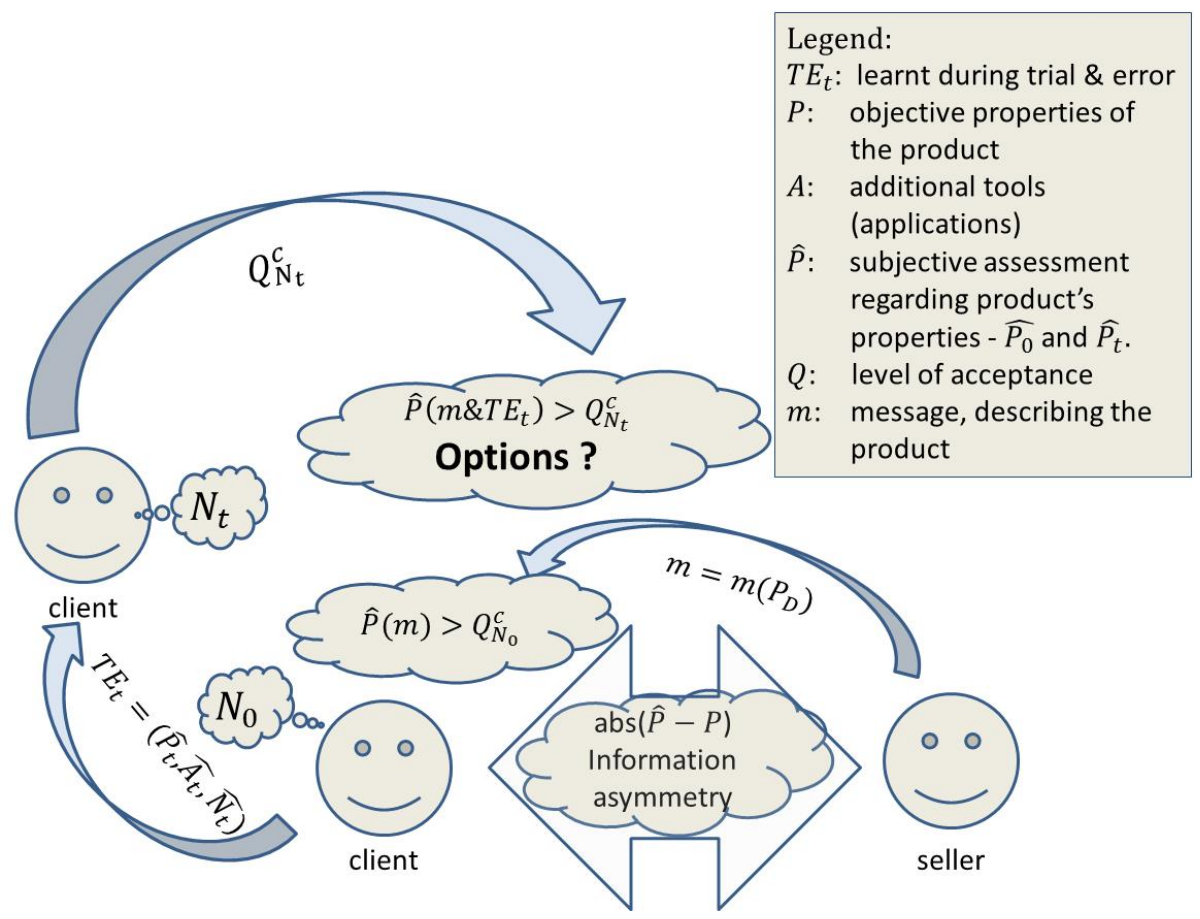

Figure 2. Illustration of the process of learning

\section{Utility}

The benefit the client $C$ will obtain by using the product $D$ to satisfy her/his needs is the value of his/her utility $U_{D}^{c}$ with respect to the product $D$.

The initial expected utility at the time of purchasing is

$$
U_{D}^{c}\left(N_{0}\right)=U\left(N_{0}, D\right),
$$

where $D=\min _{D} \widehat{P_{0}}(D)>Q_{N_{0}}^{c}$. The purchase decision is based on selecting a product, with assumed minimal propertied that satisfies the initial needs and to ensure expected utility (illustrated in Figure 3 showing the relationship between utility and gained benefit). Recall, that we assume that product price corresponds to the product's properties. The optional (possible) utility at time $t$, which is at the end of the trial and error period (the learning phase), is

$$
U_{D}^{c}\left(N_{t}\right)=U\left(N_{t}, P_{t}, A_{t}\right) .
$$

To illustrate the idea (Figure 3), let us consider the following two cases: (1) purchasing the product without misinforming warranty (the blue line) and (2) purchasing the product with an additional misinforming warranty (the red line). In both cases the client purchases the product to gain some utility, represented by the horizontal line "Utility". The client may expect that the product will bring him/her some additional benefits (see the "Expected utility"). In case (1), if the product does not satisfy the needs $\left(P>\widehat{P_{0}}\right)$, the client will be incurring losses related to the product purchase. Even if the product satisfies the initial expectations, it does not have the potential to provide higher utility than initially expected, because the client purchase decision is based on cost minimization. In case (2) the client pays extra for the warranty and is granted a risk free trial-anderror period to investigate and discover the potential of the product. This may provide very beneficial (theoretical/unlimited gain) either by discovering the product's own potential or by identifying applications that may increase dramatically the utility of the product. Also, the opportunity to replace the product during the warranty period allows the client to acquire a product with proper- 
ties that fits better his/her extended needs. Moreover, during the warranty period the client has the opportunity to adjust his/her understanding of what can be done with this type of products and what product properties are most valuable in aiming to fully utilize the product. In both cases, the client de-facto is purchasing an option, which may allow him/her to benefit beyond the initial expectations, but in the second case the client has the right to adjust his/her decision after a given trial/warranty period.

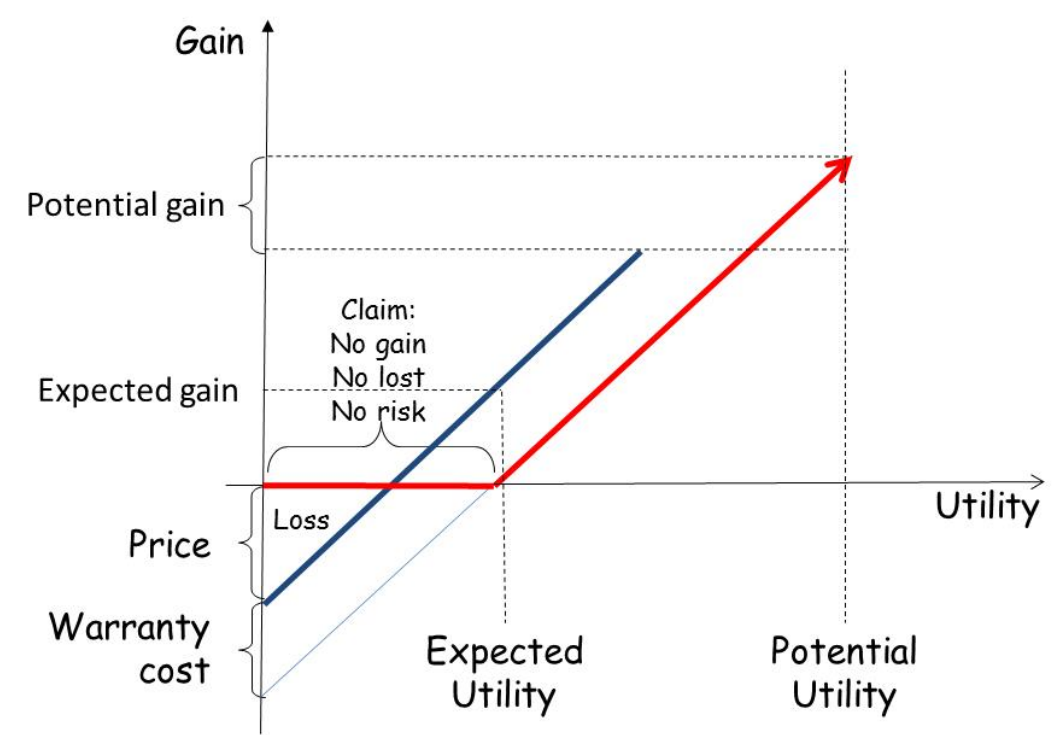

Figure 3. Illustration of the relationship between utility and gained benefit

Investing in warranty of misinforming at the time of the product purchase will provide the client with a risk free option to explore the full potential of the product including some of its extended properties and functionalities added by appropriate applications. The risk free option to obtain and explore a higher quality product, due to the added applications, allows the client to determine her/his utility and fully benefit from the product. Theoretically this benefit/gain may be infinitely large.

\section{Discussion}

In this discussion we address some open issues of the following three aspects of warranty of misinforming as an option. (1) Utility - the objectives of a customer obtaining a product, especially a smart device, always includes receiving some benefit. In principle, the actual benefit received is becoming visible only after obtaining and experimenting with the product. Warranty of misinforming, represented as a slogan "if you are not fully satisfied - money back", originally addresses the case when obtained product doesn't bring the expected utility. (2) Gain curves - acquiring the potential utility a product is able to provide may follow different processes of learning, including discovering of initially unknown product's properties, how these properties may bring value, and what are the particular requirements, such as physical parameters, bring maximal value. (3) Continuous learning and obtaining maximum value of a given product is essential. Learning includes enriching the understanding of both what the product can do for the client, but also, and equally important, what utility the client may gain by using the product. 


\section{Nominal Utility}

The examination and exploration of the product may lead to two possible outcomes:

1. The product satisfies only the initial needs and does not show any potential for solving any additional problems during the period covered by warranty of misinforming. Under this scenario, the client has two options:

- to use the warranty and to replace the product with a cheaper one, which is suitable only for the initial needs $N_{0}$ - initially "optimized" case;

- to keep the product, paying the margin between "optimal" product and the purchased one. The rationale of the second outcome is based on the following arguments: the limited trial time may not be long enough to identify fully the potential utility; the cost of the time and efforts to learn how to use the product is taken into account; and in addition, there is always a possibility that the product could turn out to be suitable for solving some extra problems that are not currently identified but could appear in the future.

2. The product shows potential to provide extra utility and it is not worth keeping it, but by exploring the warranty contract the client may acquire a higher range product that will allow him/her to benefit from solving an extended set of problems.

\section{Variety of Gain Curves}

Learning during the warranty period may progress in two directions answering to the following two questions:

- What are the properties of the product and how one can use the product, so that the properties are fully utilized?

- What are the additional problems in the costumer's domain area, such that the product is suitable to address them - identification of new problems to try to solve with the product, i.e., overcoming the initial bias regarding the suitability of the product? In this case learning allows adjusting the client's initial understanding on the idea of "perfect" product.

The actual gain of product utilization follows the learning progress and different learning curves lead to different gain curves.

\section{Importance of Learning}

The learning progress is the main key in acquiring the utility, potentially embedded in the product. Learning is usually done through trial and errors approach, because the customer has to learn how to use the product in order to address his/her own problems. Some technical assistance during the learning period is not only possible, but desirable, but a major effort has to be invested by the client to avoid misleading, misinterpretation and, and in general, misinforming.

\section{Conclusion}

In comparison with the classical use of options in finance and trade with the use of warranty of misinforming as an option, we can observe the following similarities and differences: 


\section{Similarities}

- Options and warranty of misinforming provide the client with some rights, which can be exercised only under particular circumstances.

- To obtain these rights one has to invest some resources - the price of the option/warranty of misinforming.

\section{Difference}

- In the case of options in finance and trade, if the client decides not to use the option, $\mathrm{s} / \mathrm{he}$ loses only the price paid for the option; in the case of warranty of misinforming the price, as a monetary value, is reimbursable, but the "value" obtained via investing time and efforts to explore the product - knowledge, understanding, expertise, and even recognizing a set of additional problems of interest - remains beneficial to the client in extending his/her overall skills.

- In the case of options in finance and trade the market conditions are the same for all participants and everybody who bought the option gets the same benefit - owners of an option either all use it or all do not use it. In the case of warranty of misinforming as an option some clients may use it, and others may decide not to use it.

- In the case of options in finance and trade the market conditions are the same for all participants and everybody who bought the option gets the same benefit. In the case of warranty of misinforming as an option - the cost in monetary terms is the same for everybody, but the benefit is strictly individual to the particular client.

Having in mind all of the above, we believe that considering the warranty of misinforming as an option will provide some sound theoretical and practical background and a new research direction to study, in a more formal/deeper level, the warranty of misinforming using the well-developed methods and approaches employed for studying options in finance and trade.

\section{Practical Implications}

Understanding that,

- on the one hand, the warranty of misinforming could address and remedy the negative aspects of the information asymmetry naturally affecting the communication process between sellers and buyers, and

- on the other hand, the warranty of misinforming could have a positive impact on the sellers/ buyers relationship by providing some opportunities to expand the usefulness of the considered product, is of significant benefit to the sellers.

Despite of the financial uncertainties related to the warranty of misinforming, this new understanding of misinforming may encourage the sellers to provide and promote in their businesses the risks sharing mechanisms rooted in warranties.

Also, there are significant theoretical implications of the proposed work - more systematic analysis of the warranty of misinforming could be attempted by employing theoretical, well developed and tested approaches and techniques from financial options. 


\section{References}

Christozov D., Chukova S., \& Mateev P. (2009). The risk of misinforming for competing messages. Journal of Issues in Informing Science and Information Technology, 6, 351-364. Available at http://www.informingscience.org/Publications/1064

Christozov D., Chukova S., \& Mateev P. (2011). Assessment of risk of misinforming: Dynamic measures. Interdisciplinary Journal of Information, Knowledge, and Management, 6, 163-176. Available at http://www.informingscience.org/Publications/1374

Christozov D., Chukova S., \& Mateev P. (2014). Warranty of Misinforming: An Overview, Issues in Informing Science and Information Technology, 11, 31-46. Available at http://www.informingscience.org/Publications/1978

Cohen, E. (2007). Informing and misinforming: The practice and science of using technology to inform, misinform, \& disinform. Israel Association for Information Systems 2007 Conference: Bar-Ilan University May 2007.

Cohen, E., \& Gill G. (2007). Foundations of informing science 1999-2008. Santa Rosa, CA: Informing Science Press.

Dewally, M., \& Ederington, L. (2006). Reputation, certification, warranties, and information as remedies for seller-buyer information asymmetries: Lessons from the online comic book market. Journal of Business, 79(2), 693-729.

Gackowski, Z. (2006). Quality of informing: Credibility provisional model of functional dependencies, Proceedings of $\underline{I}^{n}$ SITE $2006,6$.

Nazari, M., \& Arab, R., (2014). The influence of external signals on perceived quality and purchase intention of high involvement products (Case study: Laptop). New Marketing Research Journal, 4(2), 223241.

Option. (n.d.). In Wikipedia. Retrieved from https://en.wikipedia.org/wiki/Option

Taleb, N. (2014). Antifragile: Things that gain from disorder. Random House Trade Paperback.

Wankhade, L., \& Dabade, B. M. (2006). TQM with quality perception: A system dynamics approach. TQM Magazine, 18(4), 341-357.

\section{Biographies}

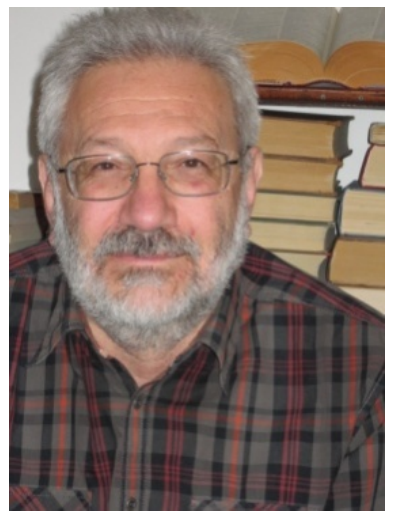

Dimitar Christozov is a Professor of Computer Science at the American University in Bulgaria. He has more than 30 years of experience in areas as computer science, quality management and information systems. He graduated in Mathematics from Sofia University "St. Kliment Ohridski" in 1979. He completed his PhD "Computer Aided Evaluation of Machine Reliability" in 1986. In 2009 he completed his D.Sc. thesis on "Quantitative Measures of Informing Quality". In ICTT "Informa" (1986-1993) Dr. Christozov was involved in establishing the national information network for technology transfer and conducted research in the areas of technologies assessment, integral quality measures and information systems for quality management. In these areas he was recognized as one of the leading experts in Bulgaria. Professor Christozov has more than 100 publications as separate volumes, journal papers and papers in refereed proceedings. He is a founding member and fellow of Informing Science Institute and chair of Bulgarian Informing Science Society; and founding member of the Bulgarian Statistical Society. Currently, Dr. Christozov is a member of Executive Council of Bulgarian National Science Fund. 


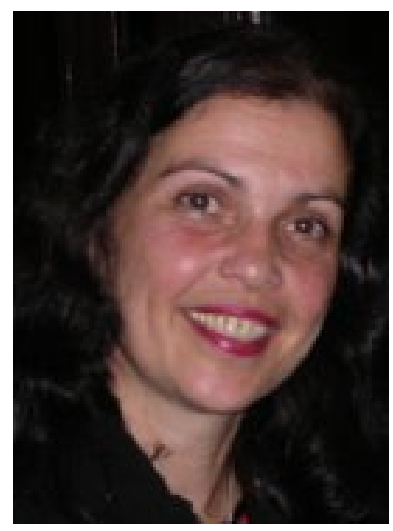

Dr. Stefanka Chukova is in Operations Research at the School of Mathematics, Statistics and Operation Research, Victoria University, Wellington, New Zealand. She has a PhD in Mathematics (concentration in Probability and Statistics) from University of Sofia, Sofia, Bulgaria. Her research interests are in applied stochastic models, warranty analysis, reliability and queuing. She has more than 80 publications and has presented papers at national and international conferences. Stefanka is an elected member of ISI.

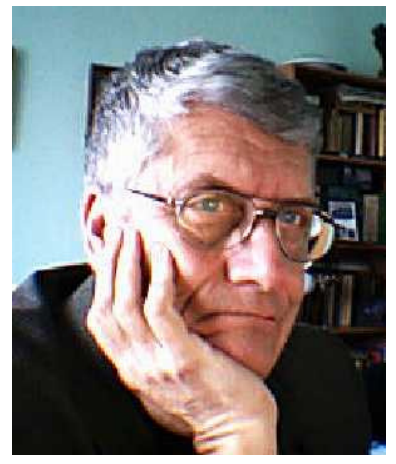

Dr. Plamen Mateev is a Professor Emeritus at the Department of Probability, Operation Research, Statistics, at Sofia University "St.Kliment Ohridski", Faculty of Mathematics and Informatics, Bulgaria. He is a member of the Statistical laboratory at the Department. Currently, he is involved in creating the Virtual Museum of Mathematics at the Institute of Mathematics and Informatics of the Bulgarian Academy of Sciences. His MSc in Mathematical Statistics is from Sofia University and his $\mathrm{PhD}$ is from Moscow State University. His research interests are in information theory, applied statistics, statistical software and applications. He has published nearly 100 papers in scientific journals and conference proceedings. He used to be a Chair of Bulgarian Statistical Society. He is a member of ENBIS, and IBS. He is a founding member of the Bulgarian Informing Science Society. 